\section{Case Reports in Ophthalmology}

Case Rep Ophthalmol 2018;9:43-48

DOI: $10.1159 / 000485888$
Published online: January 17, 2018

(C) 2018 The Author(s)

Published by S. Karger AG, Basel www.karger.com/cop

This article is licensed under the Creative Commons Attribution-NonCommercial 4.0 International License (CC BY-NC) (http://www.karger.com/Services/OpenAccessLicense). Usage and distribution for commercial purposes requires written permission.

\title{
Two Cases of Rhegmatogenous Retinal Detachment Associated with Asteroid Hyalosis
}

\author{
Yoshitaka Okuda $^{a} \quad K^{2}$ igo Kakurai ${ }^{a, b}$ Takaki Sato $^{a}$ Seita Morishita ${ }^{a, c}$ \\ Masanori Fukumoto $^{a} \quad$ Ryohsuke Kohmoto $^{a} \quad$ Mai Takagi $^{a}$ \\ Takatoshi Kobayashi $^{\mathrm{a}}$ Teruyo Kida ${ }^{\mathrm{a}}$ Tsunehiko Ikeda ${ }^{\mathrm{a}}$ \\ ${ }^{a}$ Department of Ophthalmology, Osaka Medical College, Takatsuki City, Japan; \\ ${ }^{b}$ Department of Ophthalmology, Yao Tokushukai General Hospital, Yao City, Japan; \\ 'Department of Ophthalmology, Osaka Kaisei Hospital, Osaka City, Japan
}

\section{Keywords}

Asteroid hyalosis - Rhegmatogenous retinal detachment - Posterior vitreous detachment .

Vitreous surgery

\section{Abstract}

Background: To report two cases of rhegmatogenous retinal detachment (RRD) associated with asteroid hyalosis (AH). Case Presentation: Two patients presented with RRD originating from a flap tear. Case 1 involved a 62-year-old male who was found to have bullous RRD in his left eye originating from a flap tear. During vitreous surgery, a thick vitreous cortex was found to have strongly adhered to the entire retinal surface, from the center to the periphery. A bimanual method was then used in conjunction with the vitrectomy to create an artificial posterior vitreous detachment. After surgery, the retina was successfully reattached, and his corrected visual acuity (VA) improved. Case 2 involved a 70-year-old male who was found to have localized RRD in his left eye originating from a flap tear. During vitreous surgery, a thick vitreous cortex was found to have strongly adhered to the entire retinal surface. After surgery, the retina was successfully reattached, and his corrected VA improved. Conclusions: RRD associated with AH presents with stronger vitreoretinal adhesion compared to typical $R R D$, thus requiring a more complicated surgical technique to properly treat the patient. 


\section{Introduction}

Asteroid hyalosis $(\mathrm{AH})$ is a degenerative vitreous body condition first reported by Benson in 1894 [1]. AH is marked by scattered granular opacities, such as phospholipids and mucopolysaccharides in the vitreous humor, and is reportedly often associated with diabetes and high blood pressure [2]. It has been reported that eyes affected by AH characteristically have little liquefaction in the vitreous and are resistant to posterior vitreous detachment (PVD) formation [3-5]. However, and to the best of our knowledge, there have been no reported cases of eyes affected with $\mathrm{AH}$ presenting with bullous rhegmatogenous retinal detachment (RRD). In this current study, we report two cases of RRD associated with AH, as well as our examination of the clinical characteristics and the pathogenesis of RRD.

\section{Case Presentation}

Case 1

A 62-year-old male presented at a local clinic on July 25, 2013, after becoming aware of decreased visual acuity (VA) in his left eye. Upon examination, he was diagnosed with RRD and was referred to our Department 4 days later to undergo surgery. The patient's family medical history contained nothing that signified the need of any special consideration. Upon initial examination, the patient's VA was $\left(0.6 \times-4.5 \mathrm{D}=\mathrm{C}-2.5 \mathrm{DA} \times 150^{\circ}\right)$ OD and $0.01 \mathrm{p}(0.02$ $x$-3.0D) OS, and his intraocular pressure was $20 \mathrm{~mm} \mathrm{Hg}$ OD and $16 \mathrm{~mm} \mathrm{Hg} \mathrm{OS}$. In his left eye, mild cortical cataracts were observed along with numerous asteroid bodies (AB) in the vitreous cavity, and a flap tear along the retinal lattice degeneration was found at the uppertemporal side. In addition, the eye had bullous RRD from the temporal superior side, extending to the macular area (Fig. 1a). The following day, we performed a vitrectomy on the patient's left eye. After cataract surgery, the core vitreous gel was resected. The AB were concentrated slightly toward the front, and at first observation, PVD appeared to have occurred (Fig. 1b). Upon coating the retinal surface with triamcinolone acetonide, it was ascertained that some of the $\mathrm{AB}$, as well as the somewhat thick vitreous cortex, remained across the entire retina, and that the liquefied vitreous space was very small (Fig. 1c). The membranous vitreous cortex was peeled off from the macular area to the periphery using a Diamond Dust Scraper (Synergetics USA Inc., O'Fallon, MO, USA); however, a bimanual approach to separate the vitreous membrane from the retinal surface using two vitreous forceps was required due to the strength of the adhesion across the entire retina. From the equator, the adhesion was strong, even in the quadrant where the retinal lattice degeneration did not exist. Consequently, after performing pneumatic retinal replacement and endophotocoagulation, we concurrently utilized an encircling procedure using a \#240 silicone band to diminish traction of the residual vitreous gel in the periphery. The retina was then reattached, and his corrected VA improved to $\mathrm{LV}=0.6 \times \mathrm{S}-3.0 \mathrm{D}=\mathrm{C}-0.75 \mathrm{Ax} 120^{\circ}$ (Fig. 1d).

\section{Case 2}

A 70 -year-old male presented at a local clinic on January 30, 2017, after becoming aware of visual field defect in his left eye. Upon examination, he was diagnosed with RRD and was referred to our Department that same day to undergo surgery. The patient's family medical history contained nothing that signified the need of any special consideration. Upon initial examination, the patient's VA was $\left(1.5 \times--0.25 \mathrm{D}=\mathrm{C}-1.25 \mathrm{D} \mathrm{Ax} 80^{\circ}\right)$ OD and $(1.0 \mathrm{p} \times+0.5 \mathrm{D}=$ $\mathrm{C}-2.75 \mathrm{D} \mathrm{Ax} 85^{\circ}$ ) OS, and his intraocular pressure was $16 \mathrm{~mm} \mathrm{Hg}$ OD and $16 \mathrm{~mm} \mathrm{Hg}$ OS. In 


\section{Case Reports in Ophthalmology}

Case Rep Ophthalmol 2018;9:43-48

\begin{tabular}{l|l|}
\hline DOI: $10.1159 / 000485888$ & $\odot 2018$ The Author(s). Published by S. Karger AG, Basel \\
\hline
\end{tabular} www.karger.com/cop

Okuda et al.: Two Cases of Rhegmatogenous Retinal Detachment Associated with Asteroid Hyalosis

his left eye, mild cortical cataracts were observed along with numerous $\mathrm{AB}$ in the vitreous cavity. A flap tear and localized RRD were found on the upper-temporal side (Fig. 2a). Ultrasound B-mode imaging revealed incomplete PVD and no dynamics of posterior vitreous membrane (Fig. 2b). Eleven days later, we performed vitrectomy on the patient's left eye. After cataract surgery, the core vitreous gel was resected. The AB were diffusely observed in the vitreous cavity, and the liquefied vitreous space was very small (Fig. 2c). Upon coating the retinal surface with triamcinolone acetonide, it was ascertained that some of the $A B$, as well as the somewhat thick vitreous cortex, remained across the entire retina, and that there was strong adhesion across the entire retina (Fig. 2d). The membranous vitreous cortex was peeled off from the macular area to the periphery using a Diamond Dust Scraper; however, a bimanual approach was not required in this case. Consequently, after performing pneumatic retinal replacement, we performed endophotocoagulation around the retinal tear. The retina was then reattached and his corrected VA remained 1.0.

\section{Discussion}

In regard to the clinical characteristics of $\mathrm{AH}$, it is reported that numerous yellowish particles resembling stardust sprinkled in the vitreous humor known as $\mathrm{AB}$ are found, and visibility of the ocular fundus declines [6]; however, AH presenting with symptoms that are noticeable to the affected subject, such as normal myodesopsia and decreased VA, is rare. Reportedly, $\mathrm{AB}$ adhere to collagen fibers of the vitreous body and move along with eye movements, yet return to their original position when the eye is at rest [7]. It is rare for vitrectomy to be used to treat cases of $\mathrm{AH}$, although surgery is utilized in cases with complications of the ocular fundus, i.e. cystoid macular edema, epiretinal membrane, macular hole, proliferative diabetic retinopathy (PDR), and diabetic macular edema [8].

AH usually occurs in eyes in which the posterior vitreous has not detached, and in particular, cases of PDR associated with AH frequently manifest with exceedingly strong vitreoretinal adhesion and are known for being more challenging to treat surgically [9]. Mochizuki et al. [5] reported that in eyes with $\mathrm{AH}$ appearing at first observation to have undergone PVD, a vitreous cortex is found in the posterior retina, often taking on the aspect of vitreoschisis. In the two cases in this present study, it also appeared, at first observation, that PVD had occurred, although the membranous vitreous cortex had attached to the entire surface of the retina, with strong adhesion across the entire retina. Such observations are found with typical RRD, particularly in cases of severe myopia and early-onset RRD. However, although the vitreous can generally be detached relatively easily to the equator, case 1 required an artificial PVD to be created using a bimanual approach due to the strong adhesion from the posterior fundus. In both cases in this study, the vitreous body appeared to be somewhat thicker compared to typical RRD, and revealed strong vitreoretinal adhesion from the central to peripheral area, even in the quadrant in which the lattice retinal degeneration did not exist. These findings suggest that this was a posterior wall of vitreoschisis, as noted in the above-mentioned study by Mochizuki et al. [5].

With vitreous bodies that liquefy only slightly, eyes with AH rarely present with RRD. However, both cases in this study exhibited RRD, and it is conceivable that a considerable amount of liquefied vitreous flowed under the retina through the retinal tear. Thus, it is possible that the liquid humor in the aforementioned vitreoschisis in our patients was larger than in standard eyes affected by $\mathrm{AH}$. 


\section{Case Reports in Ophthalmology}

Regarding the mechanisms by which RRD can arise in eyes that have not undergone complete PVD, such as in these two present cases, it is possible that a liquid humor in the vitreoschisis existed to the periphery and that the same intense vitreoretinal traction occurred in the rim of the lattice retinal degeneration or retinal equatorial zone. However, it is also conceivable that PVD can occur even with AH. Jingami et al. [10] reported marked improvements in VA after performing vitrectomy in a patient with retinitis pigmentosa associated with $\mathrm{AH}$. Both cases in this present study had a rapid decline in VA following cataract surgery, suggesting that the shape of the vitreous body and changes in the distribution of $A B$ due to the cataract surgery were the cause of the decline in vision. In case 1 , we observed numerous $\mathrm{AB}$ in the anterior vitreous, thus suggesting the possibility that PVD occurred.

\section{Conclusion}

The findings of this study reveal that RRD associated with AH presents with stronger vitreoretinal adhesion compared to typical RRD, thus requiring a more complicated surgical technique to treat properly, such as the use of encircling procedures in addition to the vitrectomy.

\section{Acknowledgement}

The authors wish to thank John Bush for editing the manuscript.

\section{Statement of Ethics}

This case study has been approved by the Ethics Committee of the Osaka Medical College.

\section{Disclosure Statement}

There are no conflicts of interest to report for all authors.

\section{References}

1 Benson AH: Disease of the vitreous: a case of monocular asteroid hyalitis. Trans Ophthalmol Soc UK 1894;14:101-104.

2 Fawzi AA, Vo B, Kriwanek R, Ramkumar HL, Cha C, Carts A, et al: Asteroid hyalosis in an autopsy population: The University of California at Los Angeles (UCLA) experience. Arch Ophthalmol 2005;123:486-490.

3 Wasano T, Hirokawa H, Tagawa H, Trempe CL, Buzney SM: Asteroid hyalosis: posterior vitreous detachment and diabetic retinopathy. Ann Ophthalmol 1987;19:255-258.

4 Topilow HW, Kenyon KR, Takahashi M, Freeman HM, Tolentino FI, Hanninen LA: Asteroid hyalosis. Biomicroscopy, ultrastructure, and composition. Arch Ophthalmol 1982;100:964-968.

5 Mochizuki Y, Hata Y, Kita T, et al: Anatomical findings of vitreoretinal interface in eyes with asteroid hyalosis. Graefes Arch Clin Exp Ophthalmol 2009;247:1173-1177.

Hampton GR, Nelsen PT, Hay PB: Viewing through the asteroids. Ophthalmology 1981;88:669-672.

Streeten BW: Vitreous asteroid bodies. Ultrastructural characteristics and composition. Arch Ophthalmol 1982;100:969-975. 


\section{Case Reports in Ophthalmology}

\begin{tabular}{l|l}
\hline Case Rep Ophthalmol 2018;9:43-48 \\
\hline DOI: $10.1159 / 000485888$ & $\begin{array}{l}\text { ○ 2018 The Author(s). Published by S. Karger AG, Basel } \\
\text { www.karger.com/cop }\end{array}$ \\
\hline
\end{tabular}

Okuda et al.: Two Cases of Rhegmatogenous Retinal Detachment Associated with Asteroid Hyalosis

8 Feist RM, Morris RE, Witherspoon CD, Blair NP, Ticho BH, White MF Jr: Vitrectomy in asteroid hyalosis. Retina 1990;10:173-177.

-9 Ikeda T, Sawa H, Koizumi K, Yasuhara T, Kinoshita S, Tano Y: Vitrectomy for proliferative diabetic retinopathy with asteroid hyalosis. Retina 1998;18:410-414.

10 Jingami Y, Otani A, Kojima H, Makiyama Y, Yoshimura N: Post-cataract surgery visual disturbance in a retinitis pigmentosa patient with asteroid hyalosis. Case Rep Ophthalmol 2011;2:279-282.
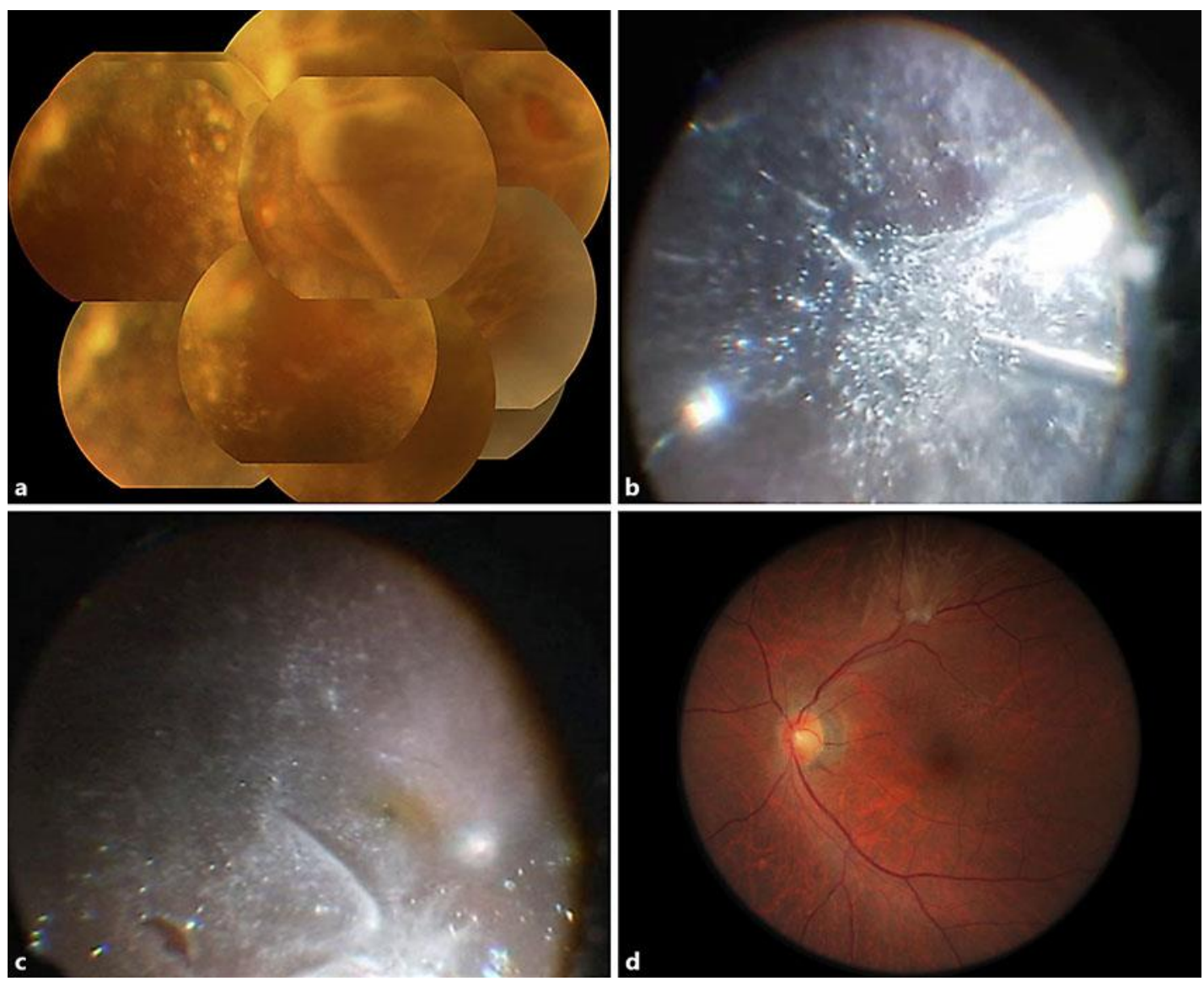

Fig. 1. A 62-year-old male patient with rhegmatogenous retinal detachment associated with asteroid hyalosis $(\mathrm{AH})$ in his left eye. a Fundoscopy image of the patient's left eye prior to surgery showing bullous retinal detachment from the temporal-superior side extending to the macular area. $\mathbf{b}$ Intraoperative image of the eye revealing asteroid bodies (AB) concentrated slightly toward the front and apparent posterior vitreous detachment. $c$ Intraoperative image of the eye after coating the retinal surface with triamcinolone acetonide showing that some of the $\mathrm{AB}$, as well as the somewhat thick vitreous cortex, remained across the entire retina. $\mathbf{d}$ Fundoscopy image of the eye after surgery showing that the retina has successfully been reattached. 


\section{Case Reports in Ophthalmology}
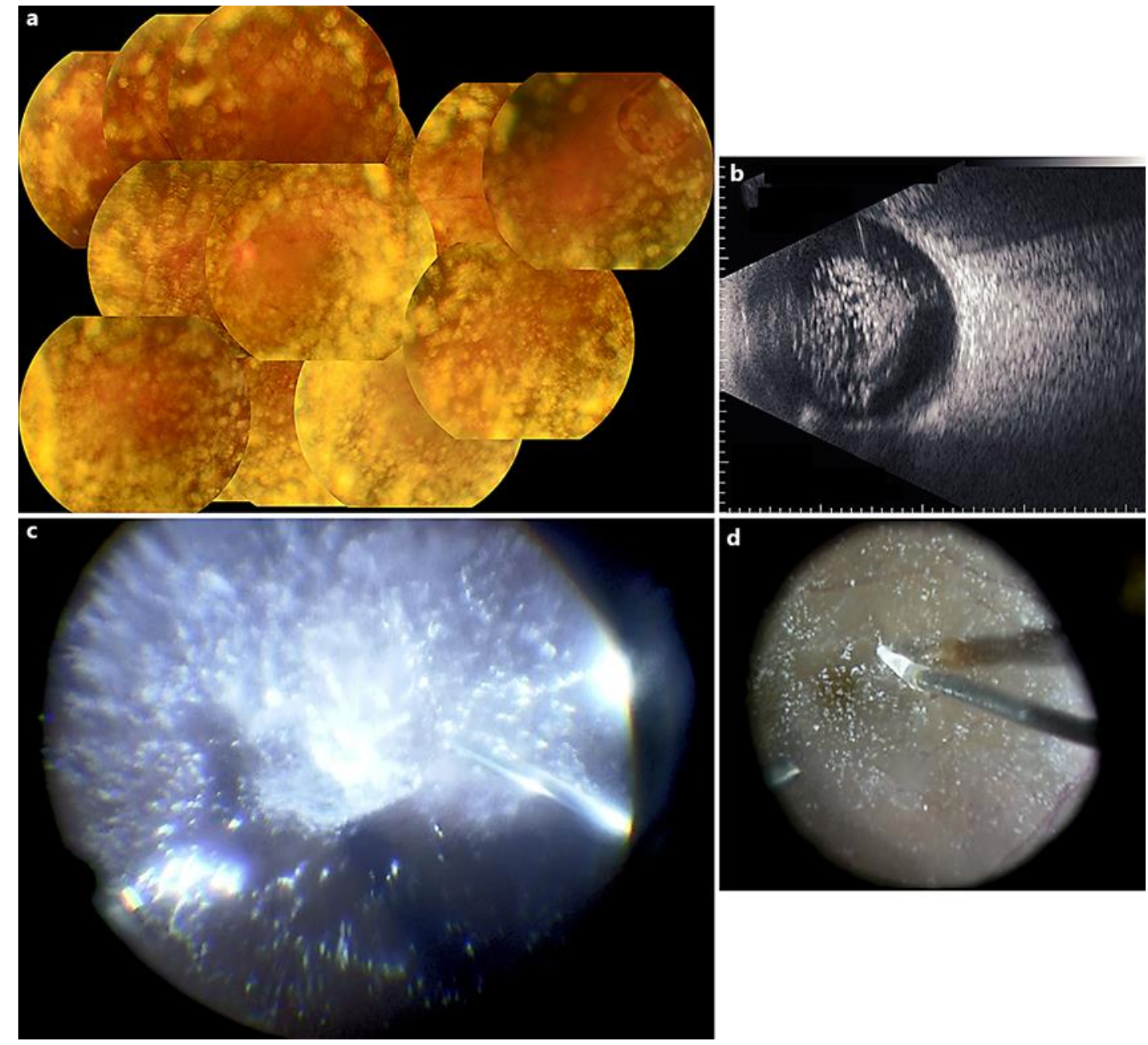

d

Okuda et al.: Two Cases of Rhegmatogenous Retinal Detachment Associated with Asteroid Hyalosis

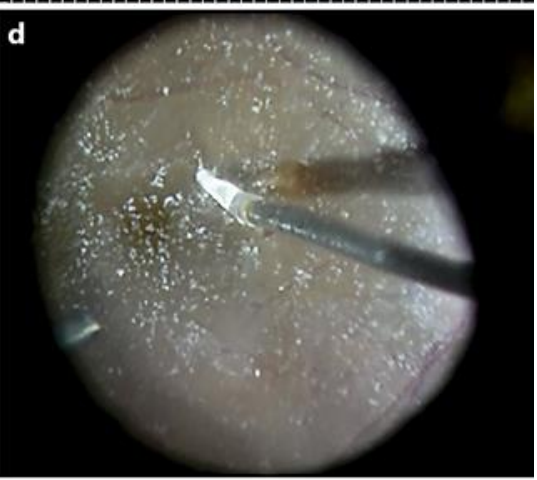

Fig. 2. A 70-year-old male patient with RRD associated with AH in his left eye. a Fundoscopy image of the patient's left eye prior to surgery showing a flap tear and localized retinal detachment at the temporalsuperior side. $\mathbf{b}$ Ultrasound B-mode image of the eye prior to surgery showing incomplete PVD and no dynamics of the posterior vitreous membrane. c Intraoperative image of the eye showing ABs diffusely in the vitreous cavity and that the liquefied vitreous space is very small. $\mathbf{d}$ Intraoperative image of the eye showing a thick vitreous cortex remaining across the entire retina, with strong adhesion across the entire retina. 